\title{
SCATTERING DYNAMICS OF EXCITONS IN ZnSe BASED SEMIMAGNETIC SEMICONDUCTORS*
}

\author{
Nguyen The Khol ${ }^{\dagger}$, W. Mac and J.A. GaJ \\ Institute of Experimental Physics, Warsaw University \\ Hoża 69, 00-681 Warszawa, Poland
}

\begin{abstract}
Resonant Raman scattering was measured for $(\mathrm{Zn}, \mathrm{Fe}) \mathrm{Se}$ and $(\mathrm{Zn}, \mathrm{Cr}) \mathrm{Se}$ with the magnetic ion mole fraction below 0.02 . LO phonons and intra-Fe ${ }^{++}$ transitions were observed. Polarization measurements versus magnetic field allowed to obtain polarization lifetimes (polariton flight times) in the subpicosecond range.
\end{abstract}

PACS numbers: 75.50.Pp, 78.30.Fs

Observation of resonant Faraday rotation of excitonic polaritons [1] has opened a new possibility of studying scattering processes of polaritons. Polarization lifetime can be measured using the exciton spin precession in an extcrnal magnetic field as a clock. The application of resonant Faraday rotation to scmimagnetic semiconductors, reported first for a Raman cascade involving intra-ion transitions in $(\mathrm{Cd}, \mathrm{Fe}) \mathrm{Se}$ [2], takes advantage of giant effective gyromagnetic factors due to exchange interaction between $\mathrm{Fe}^{++}$ions and band electron states. As a consequence, much shorter processes can be studied (down to the subpicosecond range). In this work we apply for the first time the resonant Faraday rotation method to cubic semimagnetic semiconductors $(\mathrm{Zn}, \mathrm{Fe}) \mathrm{Se}$ and $(\mathrm{Zn}, \mathrm{Cr}) \mathrm{Se}$.

Raman scattering measurements were performed in backscattering geometry using a linearly polarized $10 \mathrm{~mW}$ beam of a IIe-Cd laser. The photon encrgy of the laser light was $2.808 \mathrm{eV}$, whereas the free exciton energy in our samples was between $2.806 \mathrm{eV}$ and $2.811 \mathrm{eV}$. Our measurements were thus done under resonance conditions. The samples were placed at $2 \mathrm{~K}$ in a superconducting magnet (Faraday configuration). The spectra were analyzed as described previously [3], using a $1 \mathrm{~m}$ double grating monochromator and a GaAs photomultiplier in the photon counting regime. Polarization measurements were performed using an elastooptical modulator. In order to determine the linear polarization state of the diffused light, the polarization degree was measured twice with the analyzing system rotated by $45^{\circ}$. The two measured values constitute the real and imaginary parts of a complex polarization degree $\tilde{\mathcal{P}}$.

\footnotetext{
*Supported by the Committee for Scientific Research (grant 202239101).
}

tOn leave from Pedagogical University, Hanoi. 
In order to determine the exciton Zeeman splittings, magnetoreflectivity measurements were performed on the same samples under the same experimental conditions. In $(\mathrm{Zn}, \mathrm{Cr}) \mathrm{Se}$ two Zeeman components were resolved. In $(\mathrm{Zn}, \mathrm{Fe}) \mathrm{Se}$, where all the four usual components were visible, the splitting between the two stronger ones will be used in what follows, since the Raman resonance should be an order of magnitude stronger for those components.

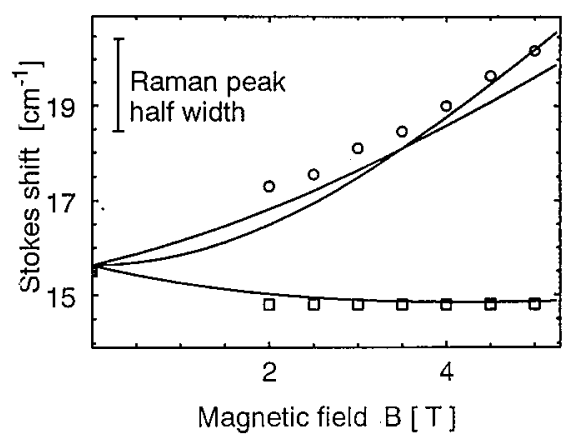

Fig. 1. $A_{1} \rightarrow T_{1}$ intra-Fe ${ }^{++}$ion transition energy in $\mathrm{Zn}_{0.985} \mathrm{Fe}_{0.015} \mathrm{Se}$ measured at $T=2 \mathrm{~K}$ vs. magnetic field. Lines are crystal field model fit with parameters: $D q=293$ $\mathrm{cm}^{-1}, \lambda=-97 \mathrm{~cm}^{-1}$ [4]. The value of $\lambda\left(-95 \mathrm{~cm}^{-1}\right.$ in Ref. [4]) has been slightly modified to fit the absolute values.

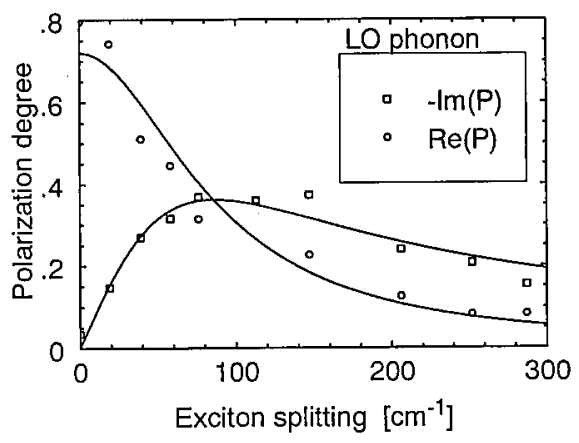

Fig. 2. Polarization degree of the LO phonon Raman line in $\mathrm{Zn}_{0.985} \mathrm{Fc}_{0.015} \mathrm{Se}$ measured at $T=2 \mathrm{~K}$ vs. exciton Zeeman splitting.

Two types of Raman spectra were observed: involving LO phonons (for all the samples) and intra-Fe $\mathrm{F}^{++}$ion excitations ( $\left.\mathrm{ZnFeSe}\right)$. Multiple and combined scattering was observed (up to 4 intra- $\mathrm{Fe}^{++}$excitations and LO phonon plus an intra-Fe $\mathrm{e}^{++}$excitation). Figure 1 shows the obtained $A_{1} \rightarrow T_{1}$ intra-ion transition encrgy versus magnetic field. The transition splits into two components consistently with a crystal field calculation [4] (two of the three components predicted by the model are too close to each other to be resolved). Similarly as in experiments on hexagonal $(\mathrm{Cd}, \mathrm{Fe}) \mathrm{Se}$ [2], Raman scattering involving intra-ion transitions 
is observed in crossed linear polarizations, unlike LO-phonon Raman scattering, where linear polarization is conserved. The results of polarization measurements for a $(\mathrm{Zn}, \mathrm{Fe})$ Se sample are shown in Fig. 2. It is easy to show that $\tilde{\mathcal{P}}$ as a function of exciton Zeeman splitting $\Delta \omega$ is a Fourier transform of the distribution of the excitonic state lifetimes. In the case of an exponential polarization decay with a lifetime $\tau, \tilde{\mathcal{P}}(\Delta \omega)$ is a complex Lorentzian with a half width $\Delta \omega_{0}=\tau^{-1}$. This result must be modified by exciton spin relaxation effects. In the simple case of exponential decay with a lifetime $\tau$ and spin relaxation time $T_{2}$ it consists in replacing $\tau$ by $\left(\tau^{-1}+T_{2}^{-1}\right)^{-1}$ and multiplying the Lorentzian by a polarization different from 1 at zero field $\mathcal{P}_{0}=T_{2} /\left(\tau+T_{2}\right)$. In order to describe the obtained polarization curves we chose temporal distributions of the form $f(t)=t^{n} \mathrm{e}^{-t / \tau}$. The corresponding polarization curves are Lorentzians to the $(n+1)$-th power: $[1 /(1-i \Delta \omega \tau)]^{n+1}$. Allowing any real (positive) value of $n$ and a spin relaxation, factor $\mathcal{P}_{0}$ gives a method of empirical description of the obtained polarization curves. Of course, the mean lifetime (proportional to the first derivative at $\Delta \omega=0$ of the imaginary part of the polarization) equals $(n+1) \tau=(n+1) / \Delta \omega_{0}$. Fits have been done for all the measurements and the results are represented in Table. The obtained lifetime values are very short and should be interpreted as times of flight of polaritons [1] (slightly modified by spin relaxation effects). The high values of $n$ obtained in some cases are evidence of non-exponential temporal distributions. This effect will be analyzed in a future publication. We were able to measure such short times due to strong Zeeman splittings occurring in semimagnetic semiconductors.

TABLE

\begin{tabular}{c|c|c|c|c|c}
\hline \hline Sample & $\begin{array}{c}\text { Magnetic ion } \\
\text { mole fraction } \\
{[\%]}\end{array}$ & Transition & $\begin{array}{c}\text { Polarization } \\
\text { lifetime }(n+1) \tau \\
{[\mathrm{ps}]}\end{array}$ & $n$ & $\mathcal{P}_{0}$ \\
\hline $\mathrm{ZnCrSe}$ & 0.5 & LO & 0.2 & 1 & 0.70 \\
\hline $\mathrm{ZnFeSe}$ & 1.5 & LO & 0.06 & 0 & 0.72 \\
& & $A_{1} \rightarrow T_{1}$ & 0.5 & 3 & 0.79 \\
$2\left(A_{1} \rightarrow T_{1}\right)$ & 0.5 & 3 & 0.54
\end{tabular}

\section{References}

[1] M. Nawrocki, R. Planel, C. Benoit à la Guillaume, Phys. Rev. Lett. 36, 1343 (1976).

[2] D. Scalbert, J.A. Gaj, A. Mauger, J. Cernogora, C. Benoit à la Guillaume, A. Mycielski, J. Cryst. Growth 101, 940 (1990).

[3] J.A. Gaj, Nguyen The Khoi, M. Nawrocki, A. Golnik, K. Lebecki, Acta Phys. Pol. $A$ 80, 409 (1991).

[4] A. Twardowski, H.J.M. Swagten, W.J.M. de Jonge, in: Proc. 19th Internat. Conf. Physics Semicond. Warszawa 1988, Ed. W. Zawadzki, Institute of Physics, Polish Academy of Sciences, Warsaw 1989, p. 1543. 